\title{
LEPTONIC DECAYS OF VECTOR MESONS: THE BRANCHING RATIO OF THE ELECTRON-POSITRON DECAY MODE OF THE PHI MESON*
}

\author{
U. Becker,$\dagger$ William K. Bertram, $\dagger$ M. Binkley, C. L. Jordan, $\dagger$ T. M. Knasel, $\dagger$ R. Marshall, $\dagger$ \\ D. J. Quinn, M. Rohde, A. J. S. Smith, and Samuel C. C. Ting $\dagger$ \\ Deutsches Elektronen-Synchrotron, Hamburg, Germany
}

(Received 30 October 1968)

\begin{abstract}
An experiment designed to measure the branching ratio of the phi meson to $e^{+} e^{-}$was performed at DESY by measuring phi production and phi decay to $e^{+} e^{-}$with the same apparatus. The results of this experiment, based on $10^{4} \varphi \rightarrow K^{+} K^{-}$events and $41 \varphi \rightarrow e^{+} e^{-}$ events on carbon, yield a branching ratio of $(2.9 \pm 0.8) \times 10^{-4}$. This result is compared with various theoretical predictions.
\end{abstract}

At the DESY 7.5-GeV electron synchrotron, experiments are in progress to investigate the leptonic decays of vector mesons. ${ }^{1}$ We report here a measurement of the branching ratio

$$
B R=\Gamma\left(\varphi \rightarrow e^{+} e^{-}\right) / \Gamma(\varphi \rightarrow \text { all })
$$

from which the coupling constant $\gamma_{\varphi}{ }^{2}$ can be obtained directly. To obtain (1), the rates of the two reactions

$$
\gamma+\mathrm{C} \rightarrow \mathrm{C}+\varphi, \quad \varphi \rightarrow e^{+} e^{-}
$$

and

$$
\gamma+\mathrm{C} \rightarrow \mathrm{C}+\varphi, \quad \varphi \rightarrow K^{+} K^{-}
$$

were measured under similar kinematic conditions using the same apparatus, a symmetric double-arm magnetic spectrometer which has been described previously. ${ }^{2}$

To first order, both the Bethe-Heitler (BH) and Compton diagrams contribute to the reaction

$$
\gamma+\mathrm{C} \rightarrow \mathrm{C}+e^{+}+e^{-},
$$

For the case in which the lepton pair $e^{+} e^{-}$has an invariant mass $M$ different from that of a vector meson, the Compton term has been estimated and measured ${ }^{3}$ to contribute less than a few percent to (4) for pair production angles $\theta \leqslant 7^{\circ}$. Therefore, for small pair production angles $\mathrm{Re}-$ action (4) can be used as a sensitive test of quantum electrodynamics at small distances. ${ }^{4}$ As first suggested by Berman and Drell, ${ }^{5}$ however, the Compton contribution will be greatly enhanced if $M$ is equal to the mass of a vector meson ( $\rho$, $\omega, \varphi)$; hence, (4) can also be used to study the leptonic decays of vector mesons.

For a measurement of the branching ratio (1) to be meaningful, three major sources of error must be reduced or avoided:

(a) $\mathrm{BH}$ background in (2) must be kept small since its subtraction contributes to (1) a statistical uncertainty as well as an uncertainty caused by lack of precise knowledge of inelastic carbon form factors. At symmetry, for a given pair invariant mass $M \simeq 2 p \theta$ ( $p$ is the momentum of the electron and positron, $\theta$ the angle of each with respect to the beam), the $\mathrm{BH}$ counting rate varies roughly as $\theta^{-6}$, whereas the $\varphi \rightarrow e^{+} e^{-}$pair rate varies as $\theta^{-3}$. Therefore,

$$
\left(\varphi \rightarrow e^{+} e^{-}\right) / \mathrm{BH} \sim \theta^{+3}
$$

and the relative $\mathrm{BH}$ background can be reduced by observing $e^{+} e^{-}$at large angles.

(b) To obtain an accurate value of the branching ratio (1), both the $\varphi$ production cross section and the $\varphi$ polarization must be known over the kinematic region for which $e^{+} e^{-}$pairs are accepted.

(c) The process $\rho \rightarrow e^{+} e^{-}$contributes to the measured $e^{+} e^{-}$at the $\varphi$ mass. The systematic errors in this subtraction can be reduced if $\rho$ production and the decay $\rho \rightarrow e^{+} e^{-}$are measured in the same apparatus.

On the basis of considerations (a), (b), and (c) above, the experimental arrangement was chosen to minimize systematic errors as follows:

First, the largest central pair opening angles compatible with a reasonable counting rate were chosen, namely $22-30^{\circ}$. The uncertainty in the carbon form factor then introduced an error $\simeq 5 \%$ in the $\mathrm{BH}$ subtraction.

Second, for this experiment, systematic errors caused by the $\varphi$ cross-section measurement were reduced by measuring both (2) and (3) with the same apparatus. ${ }^{6}$ The normalization uncertainty in the production was reduced to $\pm 16 \%$. Measurement of (3) shows that $\varphi$ production at forward angles proceeds via diffraction and that the $\varphi$ is completely transversely polarized. As shown in Fig. 1(a), the decay angular distribution $W\left(\theta^{*}\right)$ in the $\varphi$ rest system is determined to be, for $K$ pairs,

$$
W_{K K}\left(\theta^{*}\right)=(3 / 8 \pi) \sin ^{2} \theta^{*}
$$



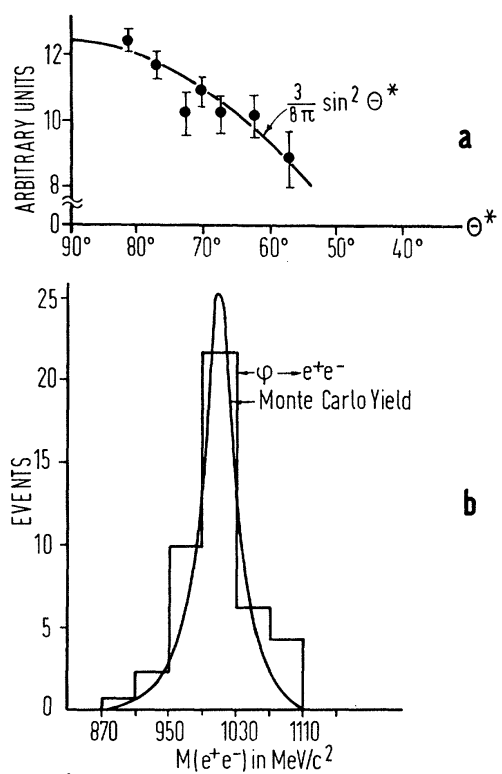

b

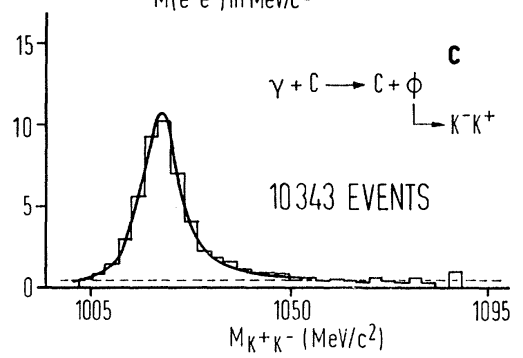

FIG. 1. (a) The angular distribution of the decay kaons in the $\varphi$ rest system. As seen, the data agree well with the distribution function Eq. (6) (solid curve). (b) The measured $\varphi \rightarrow e^{+} e^{-}$spectrum. BH background has been subtracted and all corrections made. The spectrum is in agreement with the expected $e^{+} e^{-}$mass distribution from the Monte Carlo program (solid curve). See text for details. (c) Kaon-pair invariantmass spectra at $\langle\rho\rangle=5.2 \mathrm{GeV}$. The ordinate is the cross section $d^{2} \sigma / d \Omega d m(\mu \mathrm{b} / \mathrm{sr}$ nucleon $\mathrm{MeV})$, corrected for other decay modes of the $\varphi$ meson. The dashed line is the estimate of the nonresonant background contributions. The solid curve is the $\varphi \rightarrow K^{+} K^{-}$mass distribution produced via a Monte Carlo program.

from which one obtains readily that for electron pairs:

$$
W_{e e^{\left(\theta^{*}\right)}=(3 / 16 \pi)\left(1+\cos ^{2} \theta^{*}\right)}
$$

( $\theta^{*}$ is the angle in the $\varphi$ rest system between the decay products and the recoil target particle direction).

Third, the $\rho \rightarrow e^{+} e^{-}$contribution was estimated by several independent methods: (a) Both the pion pair yield at the $\varphi$ mass and the $\rho \rightarrow e^{+} e^{-}$ branching ratio were measured with the same apparatus and hence the contribution of $\rho$ under the $\varphi$ peak calculated. (b) With the knowledge of the best fit values of mass and width to the measured $\rho \rightarrow e^{+} e^{-}$spectrum, various assumptions of the Breit-Wigner mass distribution were used to extrapolate the $\rho$ spectrum under the $\varphi$ peak. The $(\rho, \varphi)$ interference contribution was calculated assuming the production amplitude at resonance to be imaginary for $\operatorname{both}^{7} \rho$ and $\varphi$; it gives a $2.5 \%$ contribution when integrated over the acceptance. The various methods yield results $(5-15 \%)$ consistent with each other and an average value of $10 \%$ was removed from the $\varphi$ peak as the most likely $\rho$ plus $(\rho, \varphi)$ interference contamination.

Finally, to ensure that our result is not biased by interference between $\mathrm{BH}$ and Compton $(\varphi$ $-e^{+} e^{-}$) terms, and at the same time to be able to measure this interference effect, ${ }^{8}$ half of the data were taken at each spectrometer polarity.

To measure the counting rate of Reaction (2), the invariant-mass distribution of $e^{+} e^{-}$was measured for $900<M_{e e}<1150 \mathrm{MeV} / c^{2}$, a total of 103 events being accumulated. The counting rate was corrected for dead time, accidentals, and beam loss in the target. The events were grouped in $40-\mathrm{MeV}$-wide mass bins using the hodoscope information. Before the events from different settings were combined to form a mass distribution, the expected $\mathrm{BH}$ yield was subtracted for each setting. The mass distribution was then formed by combining the yields of the three spectrometer settings. In the mass region $950 \leqslant M \leqslant 1070 \mathrm{MeV} /$ $c^{2}$, a total of 84 events were observed, whereas the expected $\mathrm{BH}$ yield was 43 events.

The mass spectrum is shown in Fig. 1(b). The BH background has been subtracted, and all corrections have been applied. The spectrum is in agreement with the expected $e^{+} e^{-}$mass distribution from the Monte Carlo program. The solid curve is the $\varphi$ mass distribution produced via the program which generated $\varphi$ mesons at the target. The events were weighted by the $\varphi$ production mechanism, and the $e^{+} e^{-}$pairs were then passed through the spectrometer allowing for multiple scattering and bremsstrahlung loss until they reached a particular hodoscope combination. The effects of the bremsstrahlung were twofold: It increased the width of the observed peak to $\simeq 40$ $\mathrm{MeV} / \mathrm{c}^{2}$ and it shifted the location of the peak to a lower position. The good agreement between the Monte Carlo yield and actual data shows that almost all the events are $\varphi$ and the subtraction of background is a realistic one.

The branching ratio (1) is now easily obtained by normalizing the 41 observed $\varphi \rightarrow e^{+} e^{-}$events 
to the total number of $\varphi$ produced. The latter was calculated using the production cross section on carbon (based on $10^{4}$ events) measured in the same apparatus [Fig. 1(c)]. Taking the production mechanism and polarization factors given in Eqs. (7) and (8) into account, we obtain the result

$$
\Gamma\left(\varphi \rightarrow e^{+} e^{-}\right) / \Gamma\left(\varphi \rightarrow K^{+} K^{-}\right)=(6.1 \pm 1.7) \times 10^{-4} .
$$

Using the currently accepted value ${ }^{9}$ of

$$
\Gamma\left(\varphi \rightarrow K^{+} K^{-}\right) / \Gamma(\varphi \rightarrow \text { all })=0.473
$$

we obtain the branching ratio

$$
\mathrm{BR}=\frac{\Gamma\left(\varphi \rightarrow e^{+} e^{-}\right)}{\Gamma(\varphi-\text { all })}=(2.9 \pm 0.8) \times 10^{-4} \text {. }
$$

This determination of the branching ratio can be compared with various theoretical models as follows:

(1) Following the suggestions of Sakurai, ${ }^{10}$ the predictions of the current-mixing model together with Weinberg's first sum rule are shown as solid lines and circles in Fig. 2. The radii of the circles $=\left[\frac{1}{3} m_{\rho} \Gamma(\rho-e e)\right]^{1 / 2}$ are determined from the average of our previous measurement ${ }^{1}$ and later similar results. ${ }^{11}$ The vertical lines represent our measurement of $\Gamma\left(\varphi \rightarrow e^{+} e^{-}\right)$and together with the circles they define an "allowed region" for this model. The horizontal lines represent the Orsay measurement of ${ }^{12} \Gamma(\omega \rightarrow \ell e)$ and can be seen to overlap the "allowed region," implying that the data are consistent with Weinberg's first sum rule:

$$
{ }^{\frac{1}{3}} m_{\rho} \Gamma(\rho \rightarrow e e)=m_{\omega} \Gamma(\omega \rightarrow e e)+m_{\varphi} \Gamma(\varphi \rightarrow e e) .
$$

(2) As shown in Fig. 2, the data yield a generalized mixing angle

$$
\theta=39.2_{-5.8^{\circ}}^{+5.6^{\circ}}\left\{\tan \theta=\left[\frac{m \omega(\omega \rightarrow e e)}{m_{\varphi} \Gamma(\varphi \rightarrow e e)}\right]^{1 / 2}\right\} .
$$

This is to be compared with the predictions of Oakes and Sakurai, ${ }^{10} \theta=28.2^{\circ}$, and of Das, Mathur, and Okubo, ${ }^{13} \theta=39^{\circ}$.

(3) Our determination of the partial widths $\Gamma(\rho \rightarrow e e)=7.0 \pm 1.7 \mathrm{keV}$ and $\Gamma(\varphi \rightarrow e e)=1.2 \pm 0.4$ $\mathrm{keV}$ are in good agreement with the quark-model calculations of Dar and Weisskopf, ${ }^{14} \Gamma(\rho \rightarrow e e)$ $=5.7 \mathrm{keV}$ and $\Gamma(\varphi \rightarrow e e)=0.95 \mathrm{keV}$.

We thank Dr. J. S. Trefil for his help and advice on the data analysis, Dr. J. G. Asbury and Dr. E. Coleman for help in the earlier runs, and also Dr. A. Dar and Professor V. F. Weisskopf for many useful discussions. We are grateful for

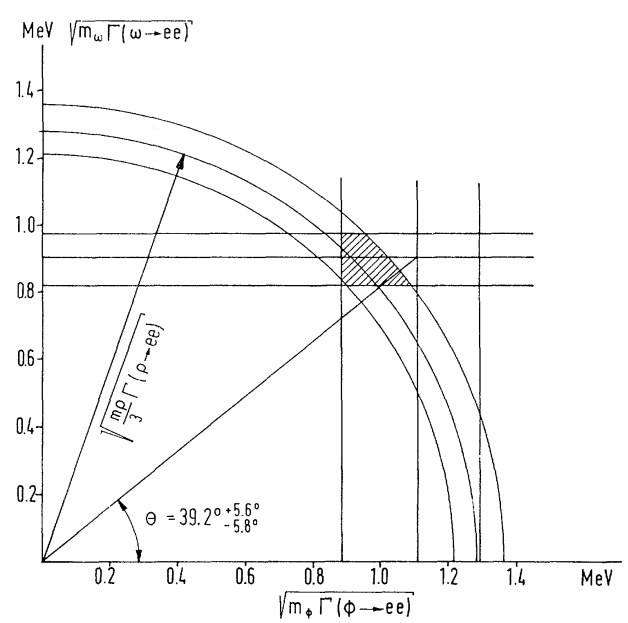

FIG. 2. Comparison of the result of this experiment with the prediction of Weinberg's first sum rule.

the support of Professor W. Jentschke, Professor M. W. Teucher, Professor V. F. Weisskopf, Professor P. Demos, Professor A. G. Hill, Professor H. O. Wuester, Professor H. Joos, and Professor G. Weber, who made this collaboration possible.

*Accepted without review under policy announced in Editorial of 20 July 1964 [Phys. Rev. Letters 13, 79 (1964)].

$\dagger$ Also Department of Physics and Laboratory for Nuclear Science, Massachusetts Institute of Technology, Cambridge, Massachusetts.

${ }^{1}$ For complete up-to-date references of the work on leptonic decays of vector mesons, see S. C. C. Ting, in the Proceedings of the Fourteenth International Conference on High Energy Physics, Vienna, September, 1968 (to be published).

${ }^{2}$ J. G. Asbury et al., Phys. Rev. 161, 1344 (1967).

${ }^{3}$ J. G. Asbury et al., Phys. Letters 25B, 565 (1967).

${ }^{4} \mathrm{H}$. Alvensleben et al., preceding Letter [Phys. Rev. Letters 21, 1501 (1968)].

${ }^{5}$ S. M. Berman and S. D. Drell, Phys. Rev. 133, B791 (1964).

${ }^{6} \mathrm{U}$. Becker et al., to be published.

${ }^{7}$ The $(\rho, \varphi)$ interference has been treated in a way similar to the approach of R. Parsons and R. Weinstein, Phys. Rev. Letters 20, 1314 (1968), and M. Davier, Stanford Linear Accelerator Center Report No. SLAC 397, 1968 (unpublished).

${ }^{8} \mathrm{U}$. Becker et al., to be published.

${ }^{9}$ A. H. Rosenfeld et al., University of California Radiation Laboratory Report No. UCRL-8030, 1968 (unpublished).

${ }^{10}$ R. J. Oakes and J. J. Sakurai, Phys. Rev. Letters 19,1266 (1967).

${ }^{11}$ We have taken $\Gamma(\rho \rightarrow e e)=6.52 \pm 0.75 \mathrm{keV}, \Gamma_{\varphi}=4.2$ $\pm 0.9 \mathrm{MeV}$, as described in Ref. 1 .

${ }^{12} \mathrm{~J}$. E. Augustin et al., in the Proceedings of the 
Fourteenth International Conference on High Energy Physics, Vienna, September, 1968 (to be published)。

${ }^{13}$ T. Das, V. S. Mathur, and S. Okubo, Phys. Rev. Let- ters 19,470 (1967).

${ }^{14}$ A. Dar and V. F. Weisskopf, Phys. Rev. Letters 26, 670 (1968).

ERR A T A

SATURATION OF THE ISOSPIN-FACTORED CURRENT ALGEBRA AT INFINITE MOMENTUM. Shau-Jin Chang, R. F. Dashen, and L. O'Raifeartaigh [Phys. Rev. Letters 21, 1026 (1968)].

There were a few typographical errors in this paper: On p. 1026, in the equation in column one, $F_{i}(\overrightarrow{\mathrm{k}})$ should be $F_{i}(\overrightarrow{\mathrm{k}})=\frac{1}{2} \tau_{i} j(\overrightarrow{\mathrm{k}})$. On the lefthand side of Eq. (8), the exponents $\exp \left(i \lambda X_{k}\right)$ should be $\exp \left(-i \lambda X_{k}\right)$. Similarly in Eq. (11), the exponents $e^{i x K_{3}}$ should be $e^{-i x K_{3}}$. Finally, the first two equations on p. 1028 should read

$$
M^{2}=g_{0}-g_{3}+g_{-}(1-\epsilon)^{-1} g_{+}, \quad\left[\overrightarrow{\mathrm{X}}, M^{2}\right]=2 i \overrightarrow{\mathrm{g}}(1-\epsilon)^{-1} \text {. }
$$

TEST OF THE EQUIVALENCE PRINCIPLE FOR UNSTABLE ELEMENTARY PARTICLES.

E. F. Beall, [Phys. Rev. Letters 21, 1364 (1968)].

The relation following Eq. (5) involving $\mu$ and $m$ should read " $\mu^{2}=m^{2}\left(1-\bar{h}^{11}\right)$."

The sentence in Ref. 2 which begins "It can also be shown..." is possibly an overstatement. What I have actually shown is that a causal, Lorentz-covariant theory is obtained if the source of the gravitational field is static, so that the field is not a wave (subject to certain approximations and to the validity of the Hilbert gauge which is used in the paper).

In Ref. 8, the expression " $\beta / n$ " should be " $\beta$ n." 